\title{
An FT-IR based instrument for measuring infrared diffuse reflectance
}

\author{
by L. Francou and P. Herve
}

Laboratoire d'Energétique et d'Economie d'Energie, Université Paris X1. chemin Desvallières 92410 Ville d'Avray, tel / fax :01.49.07.16.45m e-mail : laurent-francou@cva.u-paris10.fr

\begin{abstract}
The determination of the reflectance for directional irradiation of diffusely reflecting sample is important to improve the knowledge of thermal exchanges. It appears necessary to have some complete reflectivity measurements. Thus an infrared scattering instrument has been build and we have done measurements on several samples. The incidence angles range from $10^{\circ}$ to $80^{\circ}$. The use of a Fourier Transform spectrometer permits measurements in the $650 \mathrm{~cm}-1-8000 \mathrm{~cm}-1$ wavenumber range $(1.5 \mu \mathrm{m}-15 \mu \mathrm{m})$ and the maximal spectral resolution is $0.125 \mathrm{~cm}-1$. The instrument was tested on an alumina sample at room temperature. The wavenumber range is $1300 \mathrm{~cm}-1-8000 \mathrm{~cm}-1$.
\end{abstract}

\section{Introduction}

In many situations, some materials are under an important thermal radiation. Radiation plays an important part of the thermal transfer at elevated temperature and in the vacuum. Therefore the optical characteristics studies are important for applications as brakes and satellites. In many cases, the optical properties of opaque specimens (transmittance $\tau(\lambda)=0$ ) are characterised by the spectral reflectance for directional irradiation $\rho(\lambda)$. The spectral absorptance for directional incidence $\alpha(\lambda)$ and the emittance $\varepsilon(\lambda)$, are linked by:

$$
\begin{gathered}
\rho(\lambda)=1-\alpha(\lambda) \\
\varepsilon(\lambda)=\alpha(\lambda)
\end{gathered}
$$

Equation (1) is the Kirchhoff law and equation (2) is a statement of energy conservation.

Several methods were used to study optical characteristic [1]. The emittance or calorimetric methods [2] allow only global measurements of the various coefficient $\varepsilon, \alpha$ or $\rho$. Some experiments measure the reflected radiation in the whole hemisphere with integrating sphere [3] but cannot measure the directional reflectance. To have more information about the reflection phenomena, it appears necessary to measure the directional distribution of the reflected radiation $[4,5]$.

That's why we have build an instrument being able to measure the directional distribution of reflected radiations at several wavenumbers. The sample is at room temperature and the angle of incidence can vary from $10^{\circ}$ to $80^{\circ}$. The polarisation of the incident beam is also controlled.

\section{Experimental procedure}

The reflection method consists in focusing a monochromatic beam onto the sample and measuring the incident and the reflected flow. The rate of these two flows gives the reflectance of the sample. This rate is calculated at an angle of incidence and an angle of reflection. If the sample is motionless, the source and the detector have to move around the sample in the whole hemisphere (figure 1). The use of a Fourier Transform spectrometer [6] permits measurements in the $1.5 \mu \mathrm{m}-8 \mu \mathrm{m}$ wavelength range with a thin resolution (at $2 \mu \mathrm{m}$ the accuracy is $1 \mathrm{~nm}$ ). A hot wire gives the infrared radiation which goes through the Michelson interferometer. The signal reflected by the sample is measured by the detector. The Fourier Transformation of the signal gives the intensity of the signal for each wavelength 
but only for the signal which undergoes the Michelson. Then the furnace radiations do not interfere with the measurement. And secondly, this spectrometer is sensitive and fast because it measures all wavelength frequencies simultaneously. We have used a Nicolet 550 FT-IR spectrometer, a globar as infrared radiation source, a $\mathrm{KBr}$ beamsplitter and a MCT detector. To measure the reflectance at different polarisation states, we have used a wires grid polarizer.

For these reflection measurements the FT-IR spectrometer source radiation is directed through the interferometer where it is modulated, then directed via a parabolic off-axis mirror on the sample. The reflected beam is collected by an other parabolic off-axis mirror and focused onto the detector. The use of a metallic mirror provide us a total achromatism. The utilisation of the spectrometer as source radiation forces us to have a fixed source. Therefore, we have to use a sample holder with rotating axes. It is the same about the detector, because of the fragility of its cooling system, it could only rotate in a horizontal plane. Finally, the sample holder has two rotating axes: one vertical and one horizontal. The detector has one vertical rotating axis (figure 2).

This system of a moving sample raises a problem about the control of the polarisation of the incident beam. Indeed, as the sample has two axes of rotations, the incident beam polarisation plane changes, with the sample position. That's why we have to put the polarizer on a rotating system. Thus, the polarizer follows the plane of polarisation and the incident beam is always parallel or perpendicular to the incidence plane. The angle of rotation of the polarizer is calculated as a function of the rotation angles of the sample.

The experimental configuration forces us to work with the angles $i, \theta$ and $\varphi$ defined in figure 2 (named in the following $i_{2}, \theta_{2}$ et $\varphi_{2}$ ). But for more clearness, we have done the result with the angles $i, \theta$ and $\varphi$ of the figure 1 (named $i_{1}, \theta_{1}$ and $\varphi_{1}$ ).

The normal to sample is the zeros of the angles $i_{1}, \theta_{1}, \varphi_{1}$ and $q_{2}$.

The incident beam gives the zeros of the angles $\dot{i}_{2}$ and $\varphi_{2}$.

The relations between $i_{1}, \theta_{1}$ and $\varphi_{1}$. and $i_{2}, \theta_{2}$ and $\varphi_{2}$ are:

$$
\begin{gathered}
\cos \left(i_{1}\right)=\cos \left(\varphi_{2}\right)+\cos \left(i_{2}\right)-1 \\
\cos \left(\theta_{1}\right)=\cos \left(\theta_{2}-i_{2}\right)+\cos \left(\varphi_{2}\right)-1 \\
\sin \left(\varphi_{1}\right)=\frac{\sin \left(\varphi_{2}\right)}{\sin \left(\theta_{1}\right) \times \sin \left(i_{1}\right)}
\end{gathered}
$$

The measurement of the incident beam is a question of interest. The first solution was to measure the incident flow with a removable mirror. It appears really easier to normalize the sample spectrum with a reference spectrum. A gold mirror was placed at the sample position. The reflectance (totally specular) of this mirror is known (0.97) in the entire wavenumber range of our measurement. The accuracy of the measurement is limited by the solid angle of the detection. The mirror which focuses the reflected beam on the detector collects the flow with a $2^{\circ}$ solid angle.

This solid angle could be reduced, but for low reflecting sample it will increase the signal to noise ratio and the inaccuracy.

Measurements were carried out for:

Angles of incidence:

ranging from $0^{\circ}$ to $80^{\circ}$ with a $10^{\circ}$ step.

Angles of reflection:

In the plane of incidence:

with a $2^{\circ}$ step near the specular reflection and with a $10^{\circ}$ step further.

Out of the incidence plane:

with a $10^{\circ}$ step in the plane perpendicular tot the plane of incidence. 
Finally 351 angular configurations were carried out for each polarization state of the incident beam. For every sample, 702 spectra were acquired for a wavenumber measurements range from $1300 \mathrm{~cm}^{-1}$ to $8000 \mathrm{~cm}^{-1}$. This great number of measurements gave a complete reflectance study of the sample.

\section{Results}

The large number of results make the data reduction very long and difficult. This great quantity of measurements could seem to be useless, but it appears for the less diffusive sample that the measure near the specular reflectionare still too out spaced. The angular resolution of the directional distribution have to be thinner. We have specially looked at the spectral reflectance in the plane of incidence for several angles of incidence as a function of the wavenumber.

The directional distribution of reflected radiations (at $2 \mu \mathrm{m}$ ) got the same appearance whatever the angle of incidence; but the reflectance increases with the incidence angle (figure 3). For an incidence angle of $70^{\circ}$, alumina have a quasi specular reflectance; and it is more specular at $8000 \mathrm{~cm}^{-1}$ than at $2000 \mathrm{~cm}^{-1}$ (figure 4). The reflectivity of the nickel is presented (figure 5) at $5 \mu \mathrm{m}$ and for an incident angle of $40^{\circ}$ as function of the two angle of rotation teta and phi.

The water absorption band at $4500 \mathrm{~cm}^{-1}$ makes the measurement impossible to be performed at this wavenumber. The simple solution will be to work under a controlled atmosphere. We tried to dry the air, but the volume of the instrument was too big to allow a good measurement at this wavenumber value.

\section{Conclusion}

This work consists in the conception and the realisation of an instrument to study the reflectance of an alumina sample. The originality and the principal interest of this study is the great quantity of measurements, which could be made thanks to this instrument. The reflectivity of a sample is studied at any angle of reflection and any angle of incidence. The use of a FT-IR spectrometer allows to get measurement in a large wavenumber range and to study samples with a weak reflectance. Every kind of sample could thus be studied at room temperature. However, other measurements could be done by taking data every degree. The wavenumber range could be increased by using several detectors and beamsplitters. A cooling or heating system could be build to study the reflectance at several temperatures.

\section{REFERENCE}

[1] SACADURA (J. F.) - Proc. Colloque SFT-ISITEM-Nantes, 1990.

[2] MASUDA (H.) and HIGANO (M.) - J. Opt. Soc. Am., Vol. 2, N¹1, pp 1877-1882 1985.

[3] GINDENE (K.), KOHL (M.) and MAST (M.) - Appl. Optics, Vol.24, N¹2, 1985.

[4] AGNEW (J. T.) and Mc QUISTAN (R. B.) - J. Opt. Soc. Am., Vol. 43, №11, pp 999-1007 1953.

[5] TORRANCE (K. E.) and SPARROW (E. M.) - J. Heat. Tran., Trans. ASME, pp 223-230, 1966.

[6] MARKHAM (J. R.), SOLOMON (P. R.) and BEST (P. E.) - Rev. Sci. Instrum., Vol. 61, $N^{\circ} 12$, PP $3700-3708,1990$. 
http://dx.doi.org/10.21611/qirt.1998.036

\section{R radiation source}

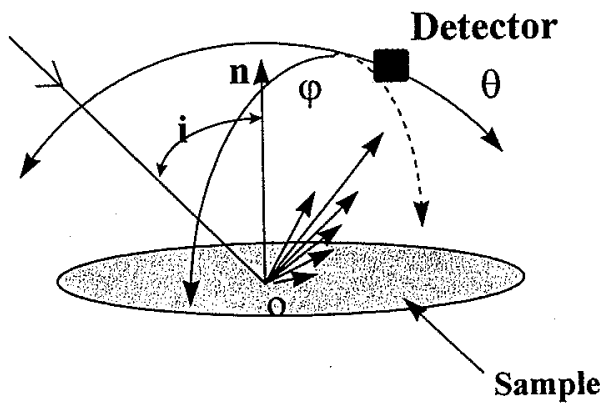

Fig. 1. Theoretical figure of the experimental procedure

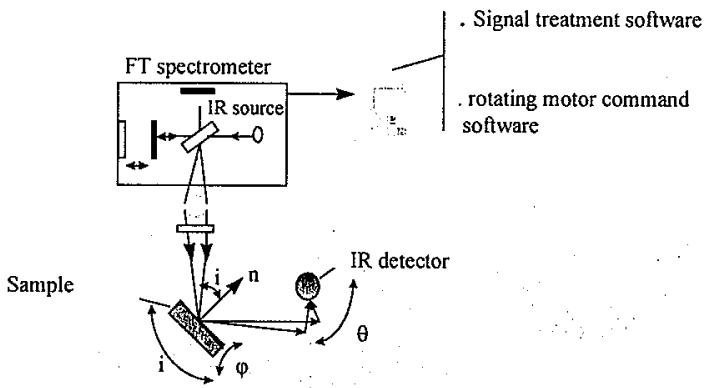

Fig. 2. Global figure of the apparatus

Polar axis graph for several angle of incidence i:

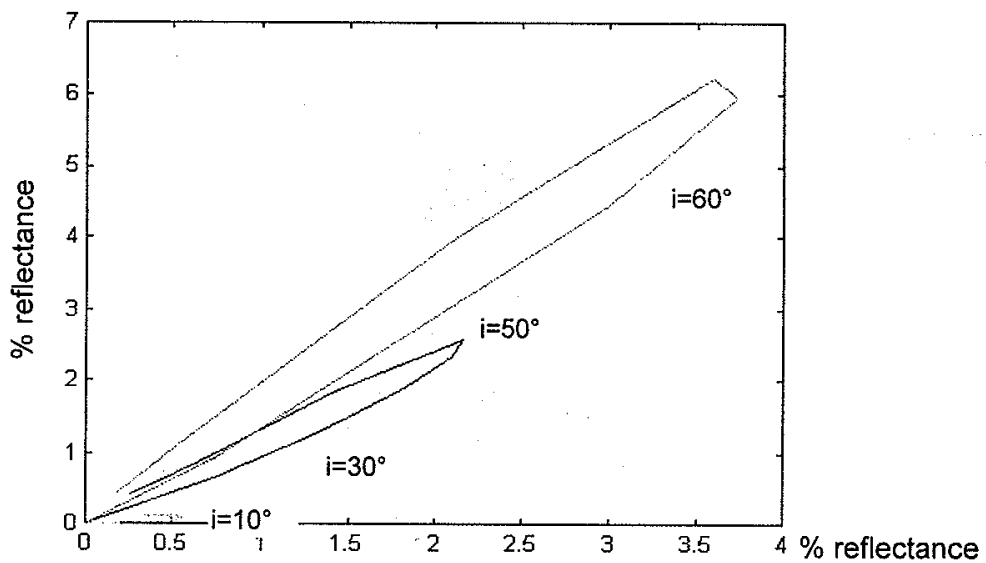

Fig. 3. Directional distribution of reflected infrared radiation on alumina sample at $\lambda=2 \mu \mathrm{m}$ 
http://dx.doi.org/10.21611/qirt.1998.036

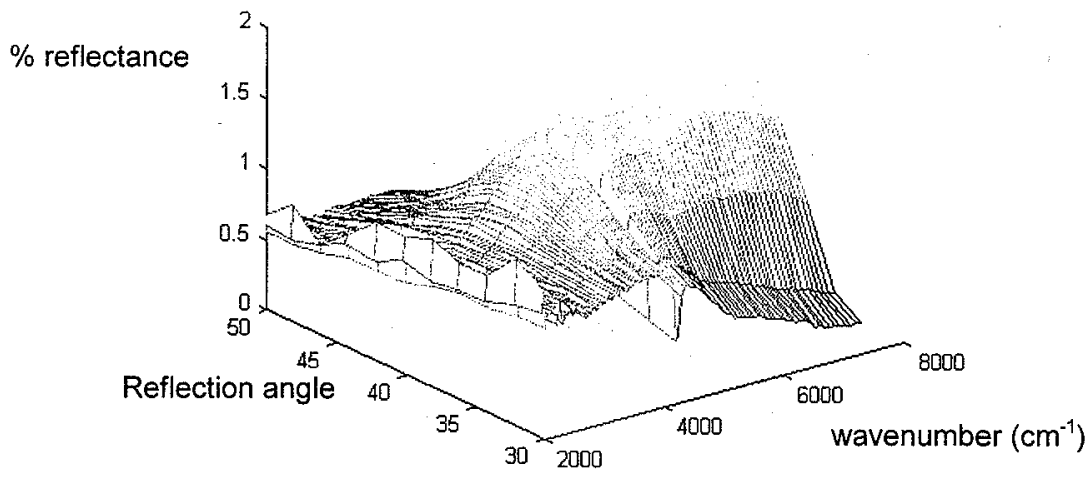

Fig. 4a. Alumina reflectance for an incidence angle: $i=40^{\circ}$

$\%$ reflectance

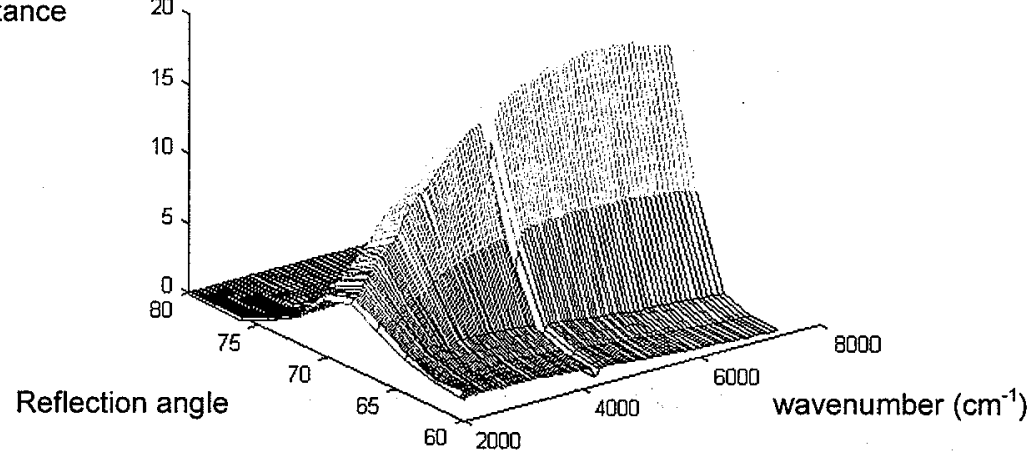

Fig. $4 b$. Alumina reflectance for an incidence angle: $i=70^{\circ}$

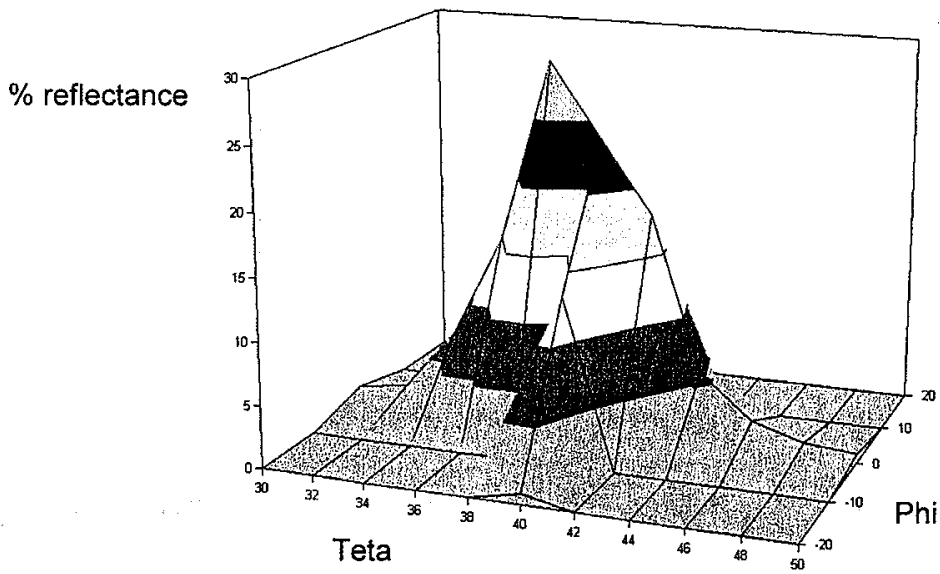

Fig. 5. Nickel reflectance at $5 \mu \mathrm{m}$ 